\title{
A non-technical overview of spatially explicit capture-recapture models
}

\author{
David Borchers
}

Received: 13 December 2009/Revised: 31 August 2010/Accepted: 10 September 2010

(C) Dt. Ornithologen-Gesellschaft e.V. 2010

\begin{abstract}
Most capture-recapture studies are inherently spatial in nature, with capture probabilities depending on the location of traps relative to animals. The spatial component of the studies has until recently, however, not been incorporated in statistical capture-recapture models. This paper reviews capture-recapture models that do include an explicit spatial component. This is done in a non-technical way, omitting much of the algebraic detail and focussing on the model formulation rather than on the estimation methods (which include inverse prediction, maximum likelihood and Bayesian methods). One can view spatially explicit capture-recapture (SECR) models as an endpoint of a series of spatial sampling models, starting with circular plot survey models and moving through conventional distance sampling models, with and without measurement errors, through mark-recapture distance sampling (MRDS) models. This paper attempts a synthesis of these models in what I hope is a style accessible to non-specialists, placing SECR models in the context of other spatial sampling models.
\end{abstract}

Keywords Spatially explicit capture-recapture . Spatial sampling - Measurement error - Capture function . Plot sampling · Distance sampling

Communicated by M. Schaub.

D. Borchers $(\square)$

Centre for Research into Ecological and Environmental

Modelling, The Observatory, Buchanan Gardens,

University of St Andrews, Fife KY16 9LZ, UK

e-mail: dlb@mcs.st-and.ac.uk

\section{Introduction}

Trapping is a common means of obtaining capture-recapture data, and one that has been used for decades, but models that explicitly incorporate the spatial component of such data are relatively new. The need for a spatial component in models arises from the fact that animals located closer to traps tend to be more likely to be captured and animals sufficiently far from the traps will certainly not be captured. Spatially explicit capture-recapture (SECR) methods incorporate the spatial information in inference.

SECR methods have found applications in a wide and growing number of areas. These include cage-trapping of possums (Efford et al. 2005), mist-netting birds (Efford et al. 2004; Borchers and Efford 2008), acoustic "trapping" of cetaceans from their vocalizations (Marques et al. 2010), acoustic "trapping" of birds from their song (Efford et al. 2009b; Dawson and Efford 2009), use of hair snares for stoats and bears together with individual identification via DNA analysis (Efford et al. 2009a; Obbard et al. 2010), visual capture-recapture of lizards (Royle and Young 2008), and camera-trapping of tigers with visual individual identification (Royle et al. 2009a, b; Royle and Dorazio 2008).

These studies have used a range of detection devices, some of which are traps while others detect without actually trapping. The devices include cage-traps (which are single-catch traps), mist-nets (a kind of multi-catch trap), acoustic detectors (both terrestrial and marine), sightings by humans, and detection by cameras. The methods are surprisingly versatile. Their basic requirements are that the location of detectors is known and that there is some means of identifying detected individuals at each detector (whatever it is) on each occasion. Actually, it turns out that, for detectors that allow detection of the same individual by 
more than one detector on a single occasion (true, e.g., for acoustic detectors, hair snares and camera traps), the methods require only a single occasion-see below for details.

These basic requirements are likely to have been met by many capture-recapture studies conducted before the advent of SECR methods, making it possible to apply SECR methods retrospectively to data from such studies.

The key additional data that SECR analyses require, over and above the data used in non-spatial capturerecapture studies, are the locations of traps at which individuals were captured. So, to develop SECR models, we need some notation for trap location. Here, it is denoted $\underline{x}$, while the animal location "centroid" is denoted $\underline{X}$. This centroid is just a means of associating a single location with an animal, it need not have biological significance. Capture probability is modeled as a function of distance from the centroid to traps. That is, capture probability depends on the distance from $\underline{x}$ to $\underline{X}(\|\underline{x}-\underline{X}\|)$. The situation is illustrated in Fig. 1.

A key question for estimating animal density is "What area do the traps effectively cover?". Because SECR models incorporate the location of traps relative to animals, they allow this question to be answered in a statistically rigorous way from the capture-recapture data themselves. Non-spatial methods rely on methods which are at least partly ad hoc to convert abundance estimates to density estimates.

Only models in which time is divided into discrete intervals called occasions are considered here. New animals may be captured, marked and released on each occasion and even captured more than once within an occasion. Traps may be of various sorts, including singlecatch traps (that hold animals for the duration of the occasion and are rendered inactive for the remainder of the occasion once they contain an animal), multiple-catch traps (that hold animals for the duration of the occasion but which remain active whether or not they contain animals)

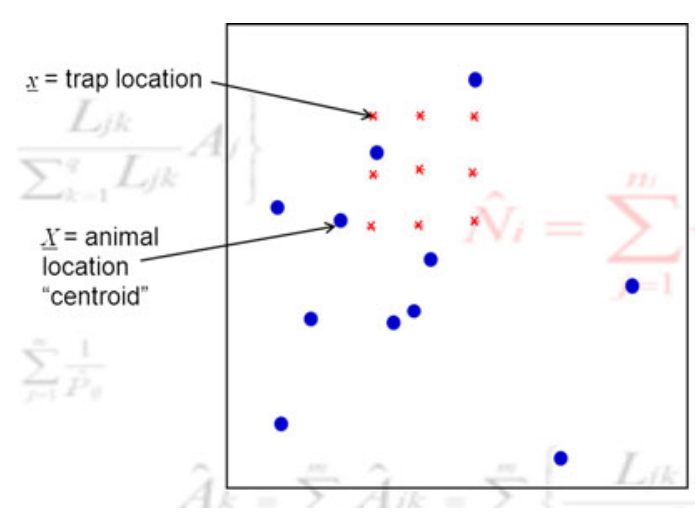

Fig. 1 Schematic representation of a spatial trapping grid relative to centroids of animal location and "proximity detectors" (traps that register animals" presence and identity, but do not hold the animal in any way). Here are some examples. Cage-traps are single-catch traps because once they hold one animal the trap can catch no others, and once it has been caught, the animal cannot be caught in any other trap before the end of the occasion. Mist-nets are multi-catch traps because they can hold any number of animals, but once an animal has been caught in a mist-net it cannot be caught in any other mist-net before the end of the occasion. Camera-traps are proximity detectors because they can detect multiple animals within an occasion, and they do not detain detected animals, which remain free to be detected by other camera-traps within each occasion.

SECR methods can be viewed as particular kinds of spatial sampling methods. I think it is therefore useful to place them in the context of other spatial sampling methods, and to this end, I very briefly review spatial sampling methods, starting with circular plot sampling in "Plot sampling". Conventional point transect sampling is summarized in "Distance sampling: point transects", followed by mark-recapture distance sampling for point transects in "Mark-recapture distance sampling" and point transect sampling with measurement errors in "Point transects with measurement error". This leads on to consideration of SECR methods proper in "Spatially explicit capturerecapture models".

For the purposes of this paper, I refer to target objects as "animals", although in reality they might be any kind of fauna, flora or inanimate object. I use the terms "captured" and "detected" interchangeably; in some contexts, it is more natural to use one rather than the other, but the models themselves make no distinction between the two.

\section{Spatial sampling models}

Plot sampling

The simplest kind of spatial sampling method is a plot sample, in which some plots comprising a subset of the survey region are sampled in such a way that every animal within the selected plot(s) is detected. The shape of the plot(s) is immaterial, and for the purposes of reviewing spatial sampling models, I consider only circular plots. Generalizing the models to plots of other shapes is straightforward but unnecessary for my purposes here. Figure 2 illustrates the situation (for simplicity, just one plot is shown).

Suppose that there are $N$ animals in the survey region and that, because $n$ of them are within the circular plot, these $n$ are detected. The outcome of the survey can be represented as $1 \mathrm{~s}$ for detected animals and 0 s for 


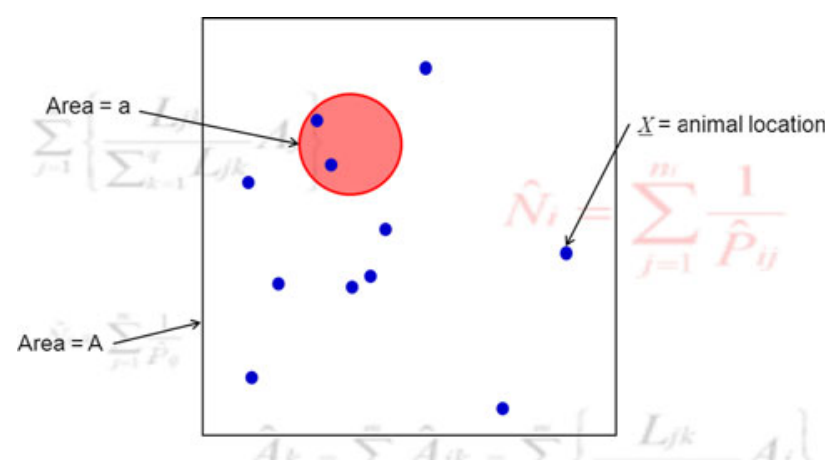

Fig. 2 Schematic representation of a circular plot sample. The survey region is represented by the outer box, with surface area $A$, the dots are animal locations and the shaded circle is a plot (with surface area a), within which all animals are detected. Animal locations are denoted $X$

undetected animals. Following Borchers et al. (2002), a useful way to model how these data arose is to use (1) a "state model" that describes probabilistically the distribution of animal locations and (2) an "observation model" that describes probabilistically how observations are obtained, given animal locations.

If we denote the $i$ th animal's location using the vector $\underline{X}_{i}$ (comprising its Cartesian coordinates in 2-dimensional space), the state model specifies the joint distribution of $\underline{X}_{1}$ to $\underline{X}_{N}: f\left(\underline{X}_{1}, \ldots, \underline{X}_{N}\right)$. The simplest such model is one in which animals are distributed independently in a region with surface area $A$ according to a 2-dimensional uniform probability density function (pdf):

$f\left(\underline{X}_{1}, \ldots \underline{X}_{N}\right)=\prod_{i=1}^{N} \frac{1}{A}$.

If the reason for the $1 / A$ term above is not obvious to you, the following analogy may help. Suppose the survey area was divided into $A$ grid cells of equal size. And suppose that, instead of being an exact location, $X_{\mathrm{i}}$ was an integer between 1 and $A$, denoting the cell in which the animal occurred. Then, if animals are equally likely to be located anywhere in the survey region, the probability that animal $i$ is located in any particular grid cell is $1 / A$, i.e. the probability of the animal having location $X_{i}$. is $1 / A$. This is true for every one of the $N$ animals. And if animals are located independently, the probability of the cells they are in being $X_{1}, \ldots, X_{N}$ is the product of the $N$ individual probabilities, as in Eq. 1 above.

Unless stated otherwise, I will assume this form for $f\left(\underline{X}_{1}, \ldots, \underline{X}_{N}\right)$ throughout this paper. In addition to it being a simple form, it has the advantage that with it the mean capture probability has an intuitive interpretation in terms of the fraction of the survey area covered by the traps (see below). This interpretation is lost with more complicated state models.
An alternative form of state model, used quite extensively in the SECR literature, that also implies a uniform pdf of animal locations, is a homogeneous Poisson process. The essential difference between that and the pdf in Eq. 1 is that, with the Poisson process, animal density $(D)$ rather than animal abundance $(N)$ is a state model parameter and $N$ is a random variable. For the purposes of this overview, I treat $N$ as fixed.

A key quantity in SECR analysis is the "effective sample area". If all animals in an area of size $A$ were captured (and none outside this area were captured) in the study, the effective sample area would fairly self-evidently be $A$. Similarly, if half the animals in an area of size $A$ were captured (and none outside this area were captured), the effective sample would be $0.5 \mathrm{~A}$. In general (when animals are uniformly distributed in the survey area), the effective sample area is $E[p] A$, where $E[p]$ is the mean capture probability in the area.

Now, if $p(\underline{X})$ is the capture probability for an animal at $\underline{X}$, then the mean capture probability of a randomly chosen animal in the population is $E[p]=\int_{A} p(\underline{X}) f(\underline{X}) \mathrm{d} \underline{X}$, where integration is over the whole survey region (with area $A$ ). If $f(\underline{X})=\frac{1}{A}$ then $E[p]=\int_{A} p(\underline{X}) \mathrm{d} \underline{X} / A=\frac{a}{A}$ where $a=$ $\int_{A} p(\underline{X}) \mathrm{d} \underline{X}$, which is readily interpreted as the effective sample area, since $a / A$ is the fraction of the survey area effectively sampled by the survey. In the case of a plot survey, $p(\underline{X})=1$ inside the plots and zero outside them (by definition), so that with a single circular plot the effective sample area is just the area of the plot.

Pursuing the grid analogy introduced above a little further and recalling that in the gridded area case $X$ is the grid cell number, then for a plot survey, $p(\underline{X})=1$ for all grid cells inside the plots (assuming here that cells are either completely inside of completely outside it). And the effective sample area $a$ is just the sum of the areas of all the grid cells falling inside the plots.

Plot surveys generate binary responses for each animal ( 1 for detected, 0 for undetected). We can represent these data, together with the observation model, state model and effective sample area as shown in Fig. 3.

\section{Distance sampling: point transects}

Distance sampling methods model detection probability as a function of distance of animals from observers. The two most common forms of distance sampling methods are point transects and line transects. In the case of line transects, detection probability is assumed to depend on the distance from a line traversed by an observer, while in the case of point transects, detection probability depends on the distance from the point at which an observer is located. We consider only the latter here, as they provide a simpler 


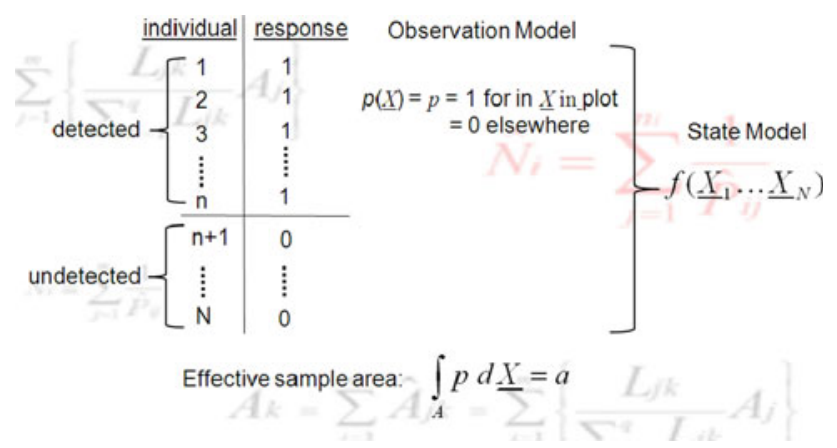

Fig. 3 Schematic representation of circular plot sample survey data, observation model, state model and effective sample area. The effective sample area is based on the uniform state model $f\left(X_{1}, \ldots\right.$, $\left.\underline{X}_{\mathrm{N}}\right)=A^{-N}$, where $A$ is the area of the survey region

extension of the circular plot survey considered above and an easier analogy when we deal with SECR models later.

Given the location ( $\underline{x}$, say) of an observer in the survey region, the probability of detecting an animal located at $\underline{X}$ is modeled as a (usually decreasing) function of the distance from $\underline{x}$ to $\underline{X}$ (i.e., of $\|\underline{x}-\underline{X}\|$ ). Figure 4 illustrates the situation.

For brevity (and since we take the observer position $x$ as known), we write the detection probability as $p(\underline{X})$ rather than $p(\underline{x}, \underline{X})$. Unlike the case with plot sampling, detection probability is not 1 everywhere inside the searched circle, but it is 1 at distance zero from the observer (i.e. at $\underline{x}$ ), and is a smooth function of distance from the observer (see Fig. 5). It need not decay to zero within any specified distance of the observer.

The effective sample area of the point transect is $a=$ $\int_{A} p(\underline{X}) \mathrm{d} \underline{X}$, with $p(\underline{X})$ decreasing smoothly as the distance from the observer $(\|x-X\|)$ increases-as illustrated in Fig. 5. Unlike the plot sampling case, $a$ will be less than the physical area of the searched region except in

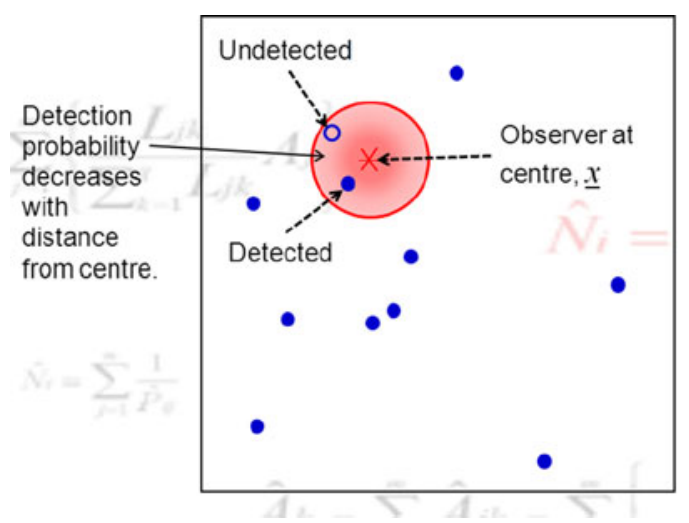

Fig. 4 Schematic representation of a point transect survey with one point. The observer is at $x$ at the center of the shaded region. Detection probability decreases away from the center (indicated by lighter shading) and not all animals in the searched region (shaded region) are detected

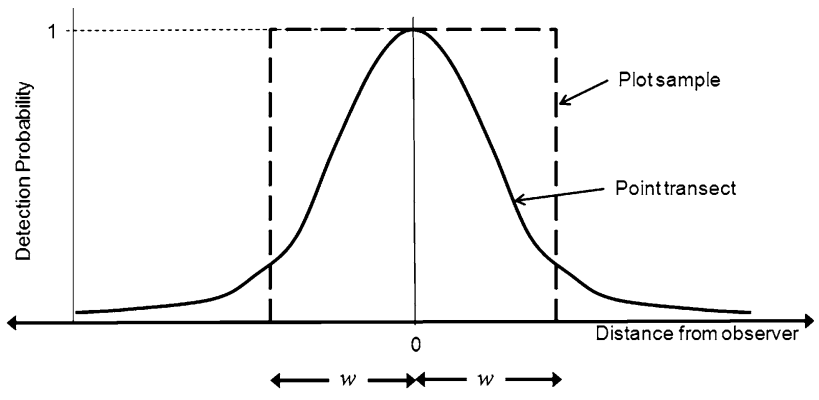

Fig. 5 Point transect detection function (solid line) in one dimension, overlaid on a plot sample detection function (dashed line). The plot center is at 0 on the horizontal axis, and this is also the location of the observer on the point transect. The radius of the plot $(w)$ is also shown

the unusual circumstance that detection probability is 1 throughout the searched region.

Whereas with plot sampling (Fig. 3), the response for each animal was binary, in the case of a point transect survey we record additional data, namely the location of each of the $n$ detected animals $\left(X_{i}, i=1, \ldots, n\right)$. In the case of point transect survey data, we therefore represent the data, observation model, state model and effective sample area for a point transect as in Fig. 6. The main differences from plot sampling (Fig. 3) are that detection probability depends on animal location and the data include animal location, as well as area searched.

Mark-recapture distance sampling

Conventional distance sampling data contain only information about the relative detection probability of animals as a function of distance, not about the absolute detection probability at any distance. In order to estimate absolute detection probability at any distance from distance data alone, they require that the detection probability at some distance is known. It is usually assumed that detection probability at distance zero (the intercept of the detection probability function) is 1 , and in many contexts, this is a reasonable assumption.

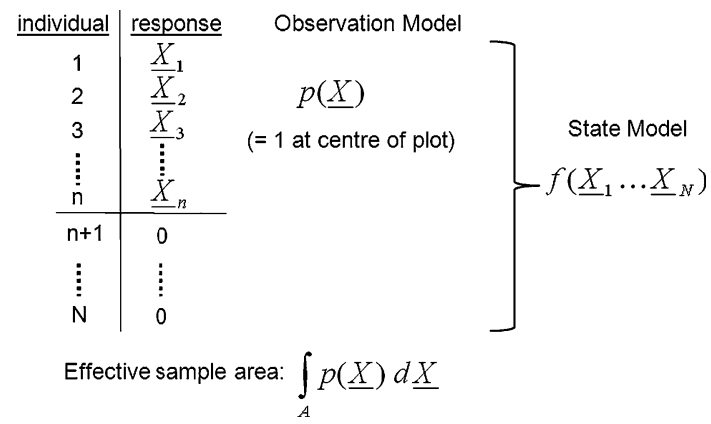

Fig. 6 Schematic representation of point transect survey data, showing observation model, state model and effective sample area. $\underline{X}_{1}, \ldots, \underline{X}_{N}$ are the locations of the $n$ detected animals 
However, in some situations, the assumption that all animals at distance zero from the observer are detected is unrealistic. This is the case for many marine surveys, where animals can be underwater and hence undetectable even though they are at distance zero. In this case, a combination of mark-recapture and distance sampling (MRDS) methods can be used (see Borchers 1996, 1998; Manly et al. 1996). These methods have been developed extensively for line transect surveys and also used on cuecounting surveys (which are like moving point transects; see Buckland et al. 2001). They have been used to a much lesser extent for stationary point transects (see Kissling et al. 2006, for an example). The underlying theory is as applicable to point transects as to line transects and it is MRDS point transect surveys that are considered here.

In this context, the survey would consist of two (or more) observers conducting independent point transect surveys with an overlap in their searched areas, with each noting the locations of detected animals. The observers could be located at the same point or different points; what is important is that their search areas overlap and that they search independently of one another. Animals detected by both observers are identified and these detections are considered to be "recaptures". Identification of recaptures is usually done on the basis of time and location-an animal detected at the same instant at exactly the same place would be regarded as the same animal. On line transect surveys, a third observer who is aware of detections by both of the independent observers is frequently used to decide whether or not detections by the two observes are recaptures. This is a bit more difficult to implement on point transect and cue-counting surveys.

A double-observer point transect survey can be thought of as a particular kind of two-occasion mark-recapture survey with individual-level covariates that include the distance of the animal from the observer $t(t=1,2)$ at $\underline{x}_{t}$ (i.e. $\left\|\underline{x}_{t}-\underline{X}\right\|$, for an animal at $\underline{X}$ ). Alternatively, it can be thought of as two occasions of a point transect survey (with detection probability $p_{t}(\underline{X})$ on occasion $t ; t=1,2$ ), with recapture information. Figure 7 illustrates a double-observer point transect survey in which the observers are located at slightly different points but with search areas that overlap. If viewed as a kind of capture-recapture survey, this figure represents two capture occasions (because there are two observers).

Unlike similar models in the capture-recapture literature for studies with individual covariates (e.g., Burnham and Overton 1978; Norris and Pollock 1996; Pledger 2000; Dorazio and Royle 2003), the MRDS likelihood includes a state model for animal locations (as in Eq. 1). This allows inferences to be made about locations of unobserved animals and hence about animal density as well as abundance; it is a spatially explicit capture-recapture model. And

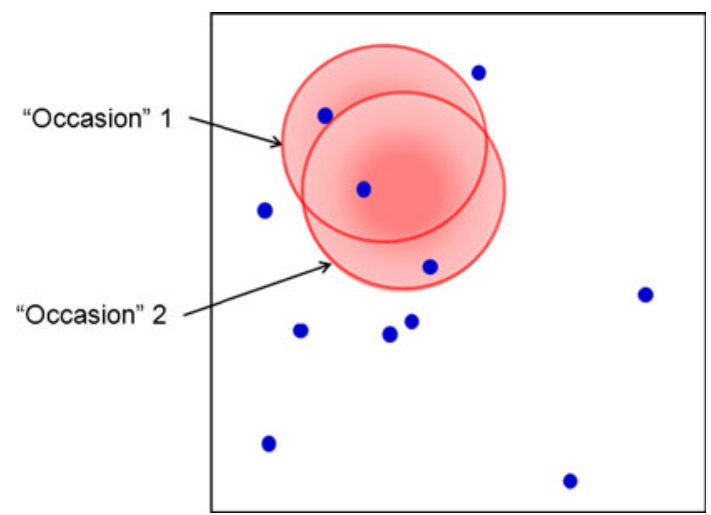

Fig. 7 Schematic representation of a mark-recapture point transect survey with two observers located at different points but with overlapping search areas (circles). "Occasion" 1 and 2 correspond to observers

unlike conventional distance sampling models, the detection function models here allow an intercept less than 1 (since, unlike conventional distance sampling, there is no constraint in the capture-recapture model on the value of the detection function at distance zero, except that it must be between 0 and 1 ).

The MRDS observation and state models and effective sample area are illustrated in Fig. 8. Here, the probability of detecting an animal at $\underline{X}$ on at least one occasion is 1 minus the probability that it is missed on all occasions. And since the probability of missing it on occasion $t$ is $\left(1-p_{t}(\underline{X})\right)$, the probability of detecting it on at least one occasion is $p_{\bullet}(\underline{X})=1-\prod_{t}\left(1-p_{t}(\underline{X})\right)$, where, $p_{t}(\underline{X})$ is the probability that observer $t$ detects the animal $(t=1,2)$.

Point transects with measurement error

Most distance sampling methods assume that distances to animals are known without error, but in some situations,

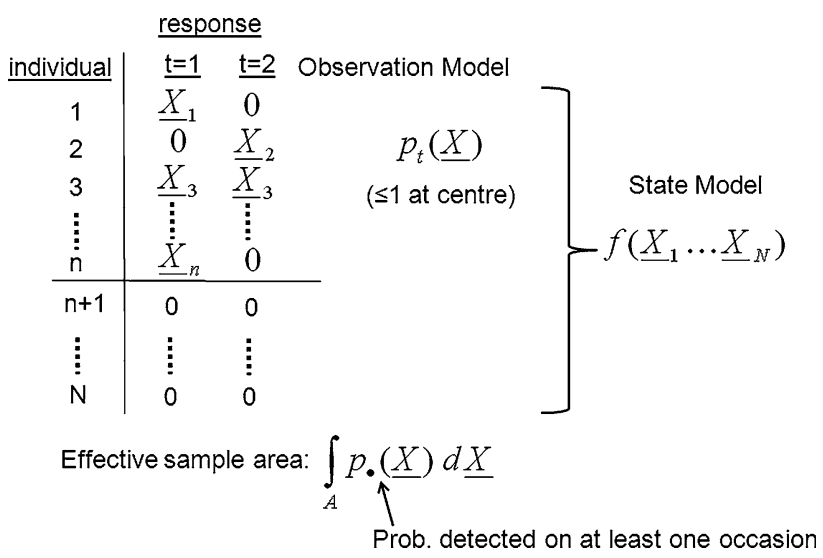

Fig. 8 Schematic representation of mark-recapture point transect survey data, showing observation model, state model and effective sample area. $p_{\bullet}(\underline{X})$ is defined in the text 
there is substantial error in measuring distances. This problem has been addressed for both line transect surveys (Alpizar-Jara 1997; Chen 1998; Chen and Cowling 2001; Marques 2004, Borchers et al. 2010) and various kinds of point transect survey, including cue-counting surveys (Hiby et al. 1989; Borchers et al. 2009, 2010). A point transect survey (i.e. one with certain detection at distance zero) in which there is measurement error is illustrated schematically in Fig. 9.

Dealing with measurement error requires an additional component for the distance sampling observation model, namely a model for the measurement error process. This is denoted $f(\underline{x} \mid \underline{X})$ where $\underline{x}$ is the recorded location (including measurement error) and $\underline{X}$ is the true location of an animal. (Note that here we use $x$ for recorded location, whereas before we used it for observer location.) Detection probability, and hence effective sample area, depends on $\underline{X}$ not $\underline{x}$. Because $\underline{X}$ is unobserved and we model the distribution of $\underline{X}$, it can be thought of as a random effect (with uniform distribution in the simple case).

The data and models from this kind of survey are illustrated in Fig. 10. Note that because we measure locations with error, our data comprise the recorded distances $\underline{x}_{1}, \ldots, \underline{x}_{n}$ instead of the true animal locations $\underline{X}_{1}, \ldots, \underline{X}_{n}$. The measurement error process is captured in the probability density function $f(\underline{x} \mid \underline{X})$ (which may need to be estimated from a subset of data for which true location is known). Detection probability still depends on the true locations, not the recorded locations. And because we are dealing with conventional distance sampling again here, we assume that detection probability at distance zero from the observer is 1 . (We are forced to assume this because conventional

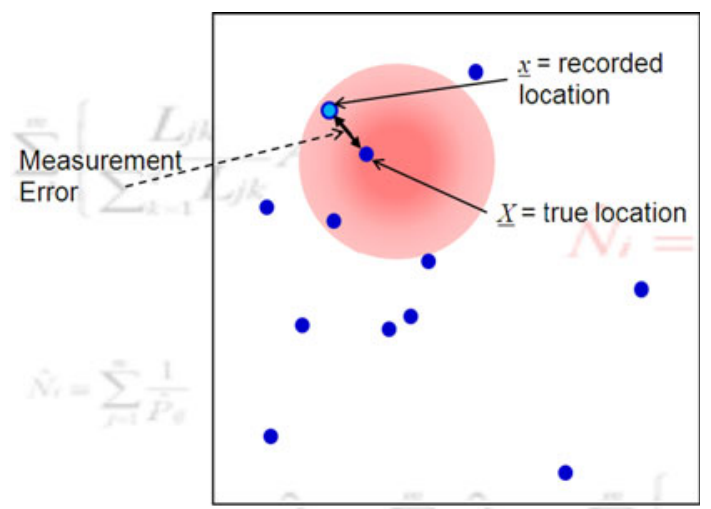

Fig. 9 Schematic representation of a point transect survey with measurement error. Here, an animal at location $X$ is recorded by the observer as being at location $\underline{x}$. (Note that here we use $\underline{x}$ for recorded location, whereas before we used it for observer location.) The measurement error is difference between the two locations (as only radial distance is normally recorded, the measurement error would usually be just the difference in radial distance to the true and recorded locations)

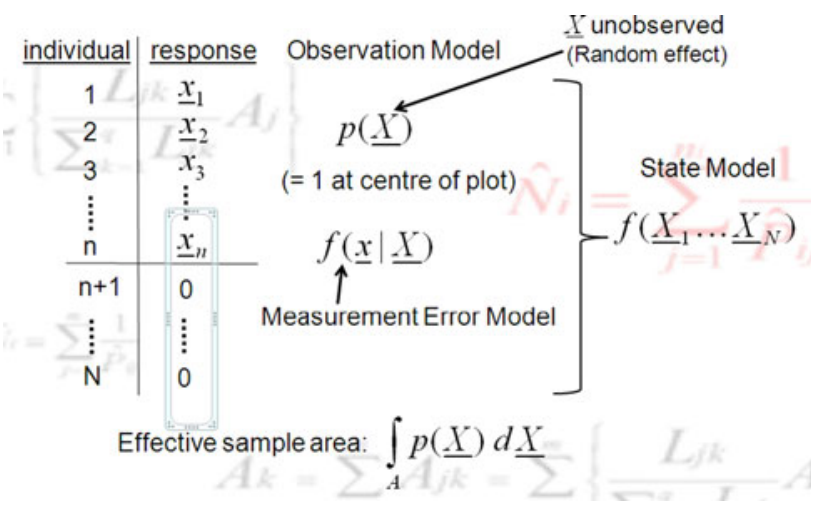

Fig. 10 Schematic representation point transect survey data with measurement error, showing observation model, state model and effective sample area. Data comprise recorded locations and the model includes a measurement error model governing how recorded locations arise from true locations

distance data are not adequate to estimate absolute detection probability anywhere, as discussed above.) Finally, because the detection probability depends on a variable that is not observed (true animal location), true animal location can be thought of as a random effect in the model.

Point transects with measurement error are of interest in this paper because it turns out that observations of location from SECR models can also be viewed as a kind of measurement error model: the location of animals' home range centers (for example) cannot be observed, but one can consider the locations of the traps at which an animal is detected as observations of the center with measurement error (more on this below).

\section{Spatially explicit capture-recapture models}

Efford (2004) proposed the first spatially explicit estimation method, based on inverse prediction. Unlike the maximum-likelihood and Bayesian estimation methods, it is not based on an explicit likelihood function and does not have the same inference foundation as these methods. It is, however, a very general estimation method, able to deal not only with all the cases that existing maximum likelihood and Bayesian methods are able to but also the single-catch trap case. In this paper, I focus on methods based on explicit likelihood functions. These are summarized briefly below.

\section{Movement-based model}

Royle and Young (2008) proposed a SECR model for estimation of horned lizard density, based on animal movement between capture occasions. Each capture occasion consisted of searching one or more rectangular plots in 
the survey region. An animal moves about its centroid at $\underline{X}$ in some random way between capture occasions, and its location at the time of capture occasion $t$ is given by $\underline{x}_{t}$ (see Fig. 11).

The model assumes that all animals that are inside the plots at the time the plots are searched are detected with the same probability (i.e. $p(\underline{x})=p$ for all $\underline{x}$ inside plots and $p(\underline{x})=0$ otherwise). The model is similar to a MRDS point transect except that detection probability is constant within the searched plot and (importantly) animals' locations change randomly between occasions. Because of this randomness, the state model requires an additional component-one to model the movement. Hence, an animal's position on occasion $t$ is modeled as a draw from the pdf $f\left(\underline{x}_{t} \mid \underline{X}\right)$. Royle and Young (2008) modeled the northing and easting components of $x_{\mathrm{t}}$ as independent normal probability density functions.

Figure 12 illustrates the data, model and effective sample area. Note that the detection probability function on occasion $t$ depends on $\underline{x}_{t}$, not $\underline{X}$. The probability that an animal at $\underline{X}$ is detected, $p(\underline{X})$, depends on the movement model $f(\underline{x} \mid \underline{X})$. As a consequence, the effective sample area also depends on the movement model. The probability that an animal at $\underline{X}$ is detected on at least one occasion is (as with the MRDS model) $p_{\bullet}(\underline{X})=1-\prod_{t}\left(1-p_{t}(\underline{X})\right)$, where $p_{t}(\underline{X})$ is the probability that the animal is detected on occasion $t$.

The probability of capture is modeled as a function of $\underline{x}_{t}$ (the animal's location on occasion $t$ ) rather than $\underline{X}$ (its location centroid). Like the effective sample area, therefore, the probability of capturing an animal with centroid $\underline{X}$ (i.e. $p(\underline{X})$ ) can be thought of as a derived property of this model rather than an explicit component.

Royle et al. (2009a) considered a variant of this model for a camera-trapping study of tigers. ${ }^{1}$ But if one treats the camera trap as a point, it has zero dimension, and the probability of an animal being at the point is consequently zero. To get around this, Royle et al. (2009a) resort to an approximation for some small area about the point. The resulting formulation is a little awkward, and in later analyses of these data, it was abandoned in favor of the more general formulation below.

So, while a model that includes in the state model a submodel for animal movement is appropriate for scenarios in which a known plot comprises the "trap", this kind of model does not generalize easily to scenarios in which traps are not clearly associated with a known area. The formulation discussed in the next section does this, and is

\footnotetext{
${ }^{1}$ Note that the "effective sample area" of Royle et al. (2009a) is not the same thing as the effective sample area of this paper. Their effective sample area is the effective area within which animals might be captured-analogous to the area of the searched region in the SECR movement model but excluding the $p(\underline{x})$ of that model.
}

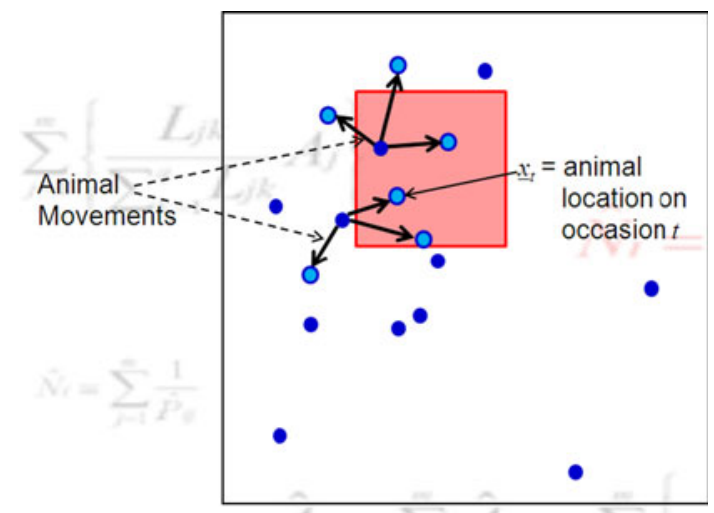

Fig. 11 Schematic representation of the horned lizard movement model, showing the movements of two animals over 3 occasions. The lighter circles with dark borders represent the animals' locations on each of the three occasions. The shaded rectangle is searched on each occasion

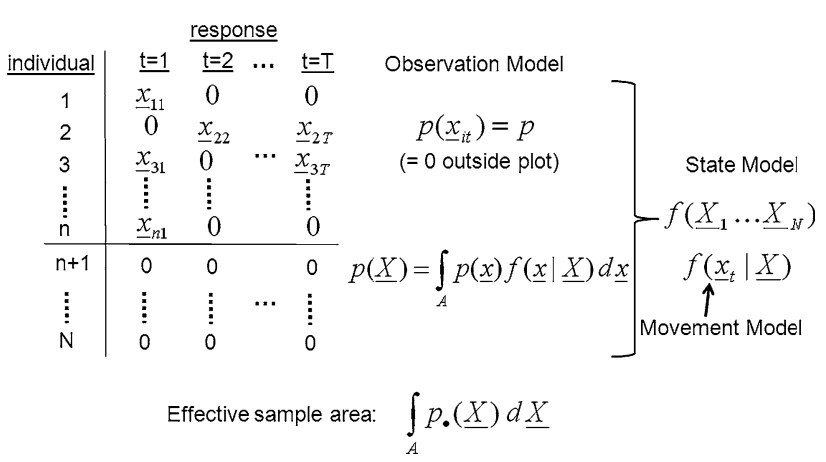

Fig. 12 Schematic representation of horned lizard movement survey data and model

also sufficiently general to include the case in which traps are associated with known plots.

\section{General model}

Efford (2004) and Borchers and Efford (2008) developed SECR models that do not require any known region to be associated with traps. This was done by treating each trap as a point (as illustrated in Fig. 1) and associating it with a general form of observation model—purposefully avoiding a model for animal movement in the state model.

While it may be the case that (part of) the reason an animal is captured is because of its movement, the general model does not attempt to separate this from the overall probability of capture - and this gives it flexibility. It can cope with situations in which there is animal movement and those in which there is none. The model is shown schematically in Fig. 13.

Notice the similarity between the measurement error observation model in Fig. 10 and the SECR observation model in Fig. 13. The only differences are (1) that the 


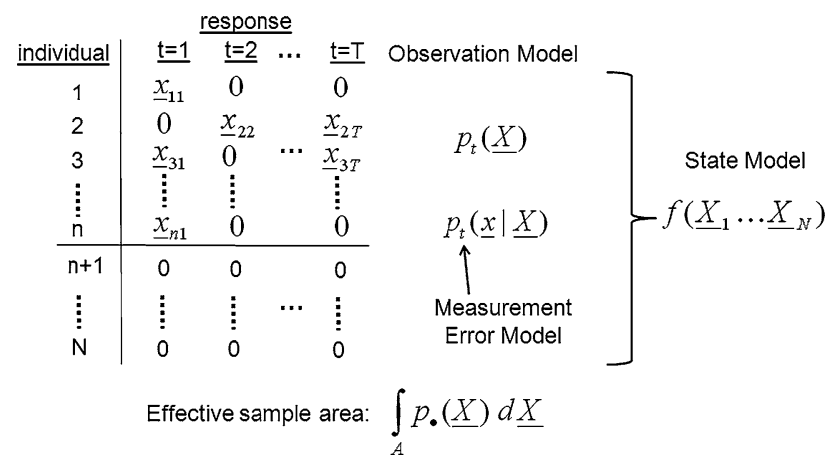

Fig. 13 Schematic representation of SECR survey data with the general model. Here, (1) $x_{i j}$, is the location of the $i$ th animal when captured on occasion $j\left(x_{i j}\right.$ is one of the trap locations since these are the only locations at which animals can be observed), and (2) the observation model includes both $\underline{x}$ and $\underline{X}$. The combined detection function for the whole array of traps on occasion $t$ is denoted $p_{\mathrm{t}}(X)$, while $p_{\mathrm{t}}(x \mid X)$ is the probability that an animal with centroid at $X$ that is detected on occasion $t$, is detected at $\underline{x}$ ( $\underline{x}$ being a trap location). The probability that an animal at $X$ is detected (by any trap) on at least one occasion is $p_{\bullet}(\underline{X})=1-\prod_{t}\left(1-p_{t}(\underline{X})\right)$

SECR observation model components have a $t$ subscript attached to them (reflecting the fact that there is more than one capture occasion), and (2) that where the measurement error observation model has a continuous probability density function $f(\underline{x \mid X)}$ (where $\underline{x}$ is any point in the plane), the SECR observation model has discrete probability density function $p(\underline{x} \mid \underline{X})$ (where $\underline{x}$ is restricted to trap location points).

One can therefore view the SECR model as a MRDS model with a particular kind of error in observing locations: a kind in which the only possible values for the observed locations are the locations of the traps.

Note that the above formulation includes the scenario in which a plot is searched, as in the case of the horned lizard example above. This can be incorporated in the general SECR model by defining the detection function appropriately. For example, if a square plot of half-width $w$ was searched, the detection function is a step function that is zero at a horizontal or vertical distance greater than $w$ from the center of the plot, and equal to an unknown parameter $p$ within horizontal and vertical distance $w$ of the plot center. Detection functions can similarly be defined to deal with plots of any shape. In their re-analysis of the cameratrap survey of tigers, Royle et al. (2009b) adopted what I have called a general SECR model rather than the movement-based SECR model of the earlier analysis (Royle et al. 2009a).

\section{SECR trap/detector varieties}

As was mentioned in the "Introduction", traps may be of various sorts. Efford et al. (2009a) identify three main kinds of trap or detector:
- Multi-catch traps. These are traps which can hold any number of animals and in which captured animals are stuck for the duration of a trapping occasion, and as a consequence, animals can only be caught in one trap on any one occasion. Mist-nets are an example.

- Single-catch traps. These are traps that can hold only one animal and which are therefore unavailable to catch other animals once they have caught one. Cage-traps are an example.

- Proximity detectors. These are "traps" that record an individual's presence but leave the animal free to be detected by other detectors within any occasion. Multiple animals may be recorded at each detector. Examples are hair snares, camera traps and acoustic detectors. (See Efford et al. 2009a for some discussion of other kinds of detector.)

The general SECR model described above accommodates all these; trap or detector type affects only the observation model, with different kinds of model being suitable for each trap or detector type-see Efford et al. (2009a, equations 5 and 6, for details).

The single-catch trap scenario is the most difficult, and of the three estimation methods developed to date (maximum likelihood, Bayesian and inverse prediction), only the inverse prediction method of Efford (2004) is currently able to deal with this case. (There is no in principle reason the other two methods cannot-it is just difficult and has not yet been done.) Fortunately, simulations by Efford et al. (2009a) indicate that the multi-catch trap model maximum likelihood estimator of density remains approximately unbiased when applied to single-catch trap data with levels of trap saturation $85 \%$ or less (i.e. when up to $85 \%$ of the single-catch traps containing an animal). This suggests it is only when trap saturation is really high that one need be very concerned about bias in density estimation when using a multi-catch trap model estimator with single-catch trap data.

The proximity detector case is interesting and likely to be particularly useful because, as pointed out by Efford et al. (2009b), it allows density to be estimated from a single capture occasion. The situation is shown schematically in Fig. 14. When animals can be detected by more than one detector in a single occasion, different detectors can play the role of occasions-with recaptures being detections of the same animal by more than one detector (as opposed to more than one occasion).

Depending on the type of proximity detector and how they are used, data from proximity detectors could either include the number of captures of an individual at each detector within an occasion or simply be binary data indicating whether or not an individual was captured at least once at each detector within an occasion. As noted 


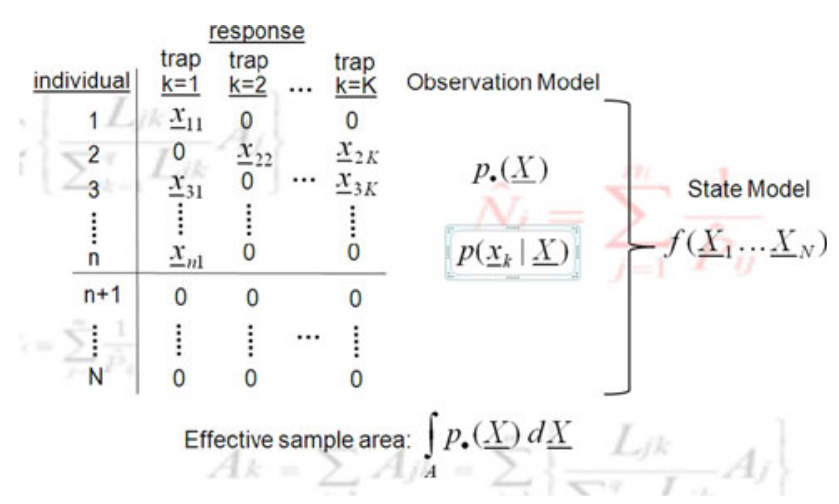

Fig. 14 Schematic representation of single-occasion SECR survey data and model. Note that data columns that corresponded to occasions in Fig. 13 correspond to traps here. The $t$ subscript has been replaced with a dot in $p_{\bullet}(\underline{X})$, which is the probability that an animal is detected by at least one trap; $p\left(x_{\mathrm{k}} \mid X\right)$ is the probability that an animal with centroid at $\underline{X}$ which is detected, is detected by the detector at $\underline{x_{\mathrm{k}}}$

by Efford et al. (2009b) and Royle et al. (2009b), the binary data are a reduced-information summary of the capture frequencies. One would therefore expect estimators based on binary data to perform less well than those based on frequency data. Efford et al. (2009b) found in a simulation study that while it did so, the difference between estimator performance in the two cases was small except in those scenarios when there was less than one recapture per individual in the population on average.

\section{Summary and discussion}

A decade ago, apparently unrelated line transect models and capture-recapture models were combined to create a model for situations in which neither approach on its own was adequate. Since then, there have been methodological advances for distance sampling when there is distance measurement error, and this has happened for the most part quite separately from advances in the development of spatially explicit capture-recapture models and methods (Royle and Dorazio 2008 being the exception).

It turns out that SECR models can be viewed as MRDS models with measurement error, and vice versa. As well as being intellectually satisfying, the marriage of MRDS and SECR models has benefits for both. For example, distance sampling detection function models have provided a basis for the development of suitable capture function models for SECR analyses (see, e.g., Efford 2004; Borchers and Efford 2008), and SECR models provide a framework for analysis of MRDS surveys with measurement error (as noted by Royle and Dorazio 2008). I expect that the exchange of ideas and methods between the two will continue, to their mutual benefit.

One area in particular which seems ripe for methodological development is the extension of SECR methods to include additional distance-related information. You can think of an MRDS point transect survey with no movement and no measurement error as the best possible kind of SECR survey-it is effectively SECR with observed animal locations. And you can think of the SECR models outlined above as MRDS point transect surveys in which none of the captured animals' locations are observed. There are a number of intermediate possibilities, including direct observation of some, but not all, animal locations, partial observation of locations (e.g., angles but not distances from traps to animals, or distances but not angles) and observation of additional variables associated with location, angle or distance (e.g., signal strength).

There has already been some development in this direction. Efford et al. (2009b) used acoustic signal strength of audially detected animals on a passive acoustic array to supplement the SECR data described above (Fig. 14), and found that using signal strength led to substantial improvements in the precision of density estimates for small arrays even though the signal strength was not calibrated against distance. I expect that, in the near future, method development will extend further into the gray area between MRDS point transects and the SECR models described above.

Other areas of likely development include the development of methods to deal with uncertain recapture identification, incorporation of models for acoustic (and other kinds of) availability, and extension to scenarios in which animals' location centroids are not stationary.

I have attempted to provide a synthesis that shows how various SECR models in the literature are related to other spatial sampling models for animal density and to each other. In trying to keep the overview accessible, I have had to restrict myself to the simpler versions of the models considered. Before leaving the subject, I should point out some of the areas in which currently available models are not quite as simple as they have appeared in my descriptions.

The first simplification I made was in considering only a uniform probability density function (pdf) for animal locations. This assumption allowed me to work with the intuitively appealing concept of effective sample area, and this helped link the various kinds of spatial sampling model. But there is no reason in principle to restrict models to have a uniform location pdf. The models of Borchers and Efford (2008), for example, include the case in which animals are distributed according to a heterogeneous Poisson process (which corresponds to a non-uniform location pdf). In this case $E[p]=\int_{A} p(\underline{X}) f(\underline{X}) \mathrm{d} \underline{X}$ does not 
have the interpretation of effective proportion of the survey area covered by the traps, instead it has a non-spatial interpretation: the expected proportion of the population that is detected or captured.

I have not considered models in which detection or capture functions depend on covariates, individual-level, survey-level or otherwise. Nor have I considered models that accommodate unobserved individual-level heterogeneity in capture/detection probability. All these kinds of model appear in the literature, together with methods such as Akaike's Information Criterion (AIC) for selecting between candidate models. I have not considered goodness-of-fit. This is an area that would benefit from further research.

Finally, I have not discussed methods of drawing inference using any of the models mentioned here. Inverse prediction methods, maximum likelihood methods and Bayesian methods have been developed and used successfully; see Efford (2004) for the inverse prediction method, Borchers and Efford (2008) and Efford et al. (2009a, b) for maximum likelihood methods, and Royle and Young (2008), Royle and Dorazio (2008) and Royle et al. (2009a, b) for Bayesian methods.

Acknowledgments I would like to thank Andy Royle for inviting me to present this work at the 2009 EURING Meeting, Tiago Marques and Len Thomas for useful feedback on an earlier draft, which led to a much improved manuscript, and to the anonymous reviewers for making suggestions that improved the accessibility and readability of the manuscript.

\section{References}

Alpizar-Jara R (1997) Assessing assumption violation in line transect sampling. PhD thesis, North Carolina State University, Raleigh

Borchers DL (1996) Line transect estimation with uncertain detection on the trackline. $\mathrm{PhD}$ thesis, University of Cape Town

Borchers DL, Efford MG (2008) Spatially explicit maximum likelihood methods for capture-recapture studies. Biometrics 64:377-385

Borchers DL, Zucchini W, Fewster RM (1998) Mark-recapture models for line transect surveys. Biometrics 54:1207-1220

Borchers DL, Buckland ST, Zucchini W (2002) Estimating animal abundance: closed populations. Springer, London

Borchers DL, Pike D, Gunnlaugsson T, Vikingsson GA (2009) Minke whale abundance estimation from the NASS 1987 and 2001 cue counting surveys taking account of distance estimation errors. North Atlantic Marine Mammal Commission Special Issue 7: North Atlantic Sightings Surveys (1987-2001), pp 95-110

Borchers DL, Marques TA, Gunlaugsson T, Jupp P (2010) Estimating distance sampling detection functions when distances are measured with errors. J Agric Biol Environ Stat. doi:10.1007/ s13253-010-0021-y

Buckland ST, Anderson DR, Burnham KP, Laake JL, Borchers DL, Thomas L (2001) Introduction to distance sampling: estimating abundance of biological populations. Oxford University Press, Oxford
Burnham KP, Overton WS (1978) Estimation of the size of a closed population when capture probabilities vary among animals. Biometrika 65:625-633

Chen SX (1998) Measurement errors in line transect surveys. Biometrics 54:899-908

Chen SX, Cowling A (2001) Measurement errors in line transect sampling where detectability varies with distance and size. Biometrics 57:732-742

Dawson DK, Efford MG (2009) Bird population density estimated from acoustic signals. J Appl Ecol 46:1201-1209

Dorazio RM, Royle JA (2003) Mixture models for estimating the size of a closed population when capture rates vary among individuals. Biometrics 59:351-364

Efford MG (2004) Density estimation in live-trapping studies. Oikos 106:598-610

Efford MG, Dawson DK, Robbins CS (2004) DENSITY: software for analysing capture-recapture data from passive detector arrays. Anim Biodivers Conserv 27:217-228

Efford MG, Warburton B, Coleman MC, Barker RJ (2005) A field test of two methods for density estimation. Wildl Soc Bull 33:731-738

Efford MG, Borchers DL, Byrom AE (2009a) Density estimation by spatially explicit capture-recapture: likelihood-based methods. In: Thompson DL, Cooch EG, Conroy MJ (eds) Modeling demographic processes in marked populations. Springer, New York, pp 255-269

Efford MG, Dawson DK, Borchers DL (2009b) Population density estimated from locations of individuals on a passive detector array. Ecology 90:2676-2682

Hiby L, Ward A, Lovell P (1989) Analysis of the North Atlantic sightings survey 1987: aerial survey results. Rep Int Whaling Comm 39:447-455

Kissling ML, Garton EO, Handel CM (2006) Estimating detection probability and density from point-count surveys: a combination of distance and double-observer sampling. Auk 123:735-752

Manly B, McDonald L, Garner G (1996) Maximum likelihood estimation for the double-count method with independent observers. J Agric Biol Environ Stat 1:170-189

Marques TA (2004) Predicting and correcting bias caused by measurement error in line transect sampling using multiplicative error models. Biometrics 60:757-763

Marques TA, Thomas L, Martin SW, Mellinger DK, Jarvis S, Morrissey RP, Ciminello C, DiMarzio N (2010) Spatially explicit capture recapture methods to estimate minke whale abundance from data collected at bottom mounted hydrophones. J Ornithol. doi:10.1007/s10336-010-0535-7

Norris JL, Pollock KH (1996) Nonparametric MLE under two closed capture-recapture models with heterogeneity. Biometrics 52: 639-649

Obbard ME, Howe EJ, Kyle C (2010) Empirical comparison of density estimates for large carnivores. J Appl Ecol 47:76-84

Pledger S (2000) Unified maximum likelihood estimates for closed capture-recapture models using mixtures. Biometrics 56:434442

Royle JA, Dorazio RM (2008) Hierarchical modeling and inference in ecology. Academic, London

Royle JA, Young KV (2008) A hierarchical model for spatial capture-recapture data. Ecology 89:2281-2289

Royle JA, Nichols JD, Karanth KU, Gopalaswamy AM (2009a) A hierarchical model for estimating density in camera-trap studies. J Appl Ecol 46:118-127

Royle JA, Karanth KU, Gopalaswamy AM, Kumar NS (2009b) Bayesian inference in camera-trapping studies for a class of spatial capture-recapture models. Ecology 90:3233-3244 